\title{
Some New Trajectory Patterns and Periodic Behaviors of Unstable Second-order Digital Filter with Two's Complement Arithmetic
}

\author{
WING-KUEN LING and KWONG-SHUM TAM \\ Department of Electronic and Information Engineering \\ The Hong Kong Polytechnic University \\ Hung Hom, Kowloon, Hong Kong, China
}

\begin{abstract}
This letter shows some counter-intuitive simulation results that for some filter parameters in the extended boundaries of the stability triangle, the state vector will converge to a periodic orbit after some iterations, no matter what the initial conditions are. Also, a new pattern, which looks like a rotated letter ' $\mathrm{X}$ ', is found. The center of the rotated letter is located at the origin and the slopes of the 'straight lines' of the rotated letter are equal to the values of the pole locations.
\end{abstract}

\section{Introduction}

It is well known that chaotic behavior may occur in a second-order digital filter with two's complement arithmetic [Chua, 1988, 1990; Galias, 1992; Kocarev, 1993, 1996; Wu, 1993; Yu, 2001]. For the characteristic equation of the all-pole linearized digital filter being $z^{2}-a \cdot z-b$, where the filter parameters $(a, b)$ are in the set $\{(a, b):|a| \leq 2$ and $b=-1\}$, the phase portrait of the state variables, using the direct method of implementation, may exhibit one of three types of trajectories, namely the type I, II, and III trajectories. The type I trajectory corresponds to a single rotated ellipse. For the type II trajectory, there are more than one rotated and translated ellipses. The type III trajectory is characterized by an elliptical fractal pattern [Chua, 1998, 1990; Galias, 1992; $\mathrm{Wu}, 1993 ; \mathrm{Yu}, 2001]$. The state vector $\mathbf{x}(k)$ is periodic for the type I and type II trajectories, but aperiodic for the type III trajectory. When the filter parameters $(a, b)$ are in the set $\{(a, b):|a|>2$ and $b=-1\}$, the phase portrait exhibits a dense countable set of discontinuous lines, and shows a random-like chaotic 
pattern, illustrating that the state vector $\mathbf{x}(k)$ is aperiodic. One typical example is that when $a=3$ and $b=-1$, the system will correspond to the well known Arnold-Sinai cat map [Kocarev, 1993]. When the filter parameters $(a, b)$ are in the set $\{(a, b):(b=a+1$ and $|b|>1)$ or $(b=-a+1$ and $|b|>1)\}$, there are some straight lines on the phase portrait [Kocarev, 1996]. The state vector $\mathbf{x}(k)$ is aperiodic for most of the initial conditions.

According to the existing literature [Kocarev, 1993, 1996], one might expect that when the filter parameters $(a, b)$ are on the extended boundaries of the stability triangle, $\quad$ that $\quad$ is, $\quad\{(a, b):(|a|>2$ and $b=-1)$ or $(b=a+1$ and $|b|>1)$ or $(b=-a+1$ and $|b|>1)\}$, then the state vector $\mathbf{x}(k)$ is aperiodic for most of the initial conditions because one of the eigenvalues of the linearized digital filter has magnitude greater than one. However, we show some counter-intuitive simulation results in section 2 that for some filter parameters $(a, b)$ on the extended boundaries of the stability triangle, the state vector $\mathbf{x}(k)$ will converge to a periodic orbit after a number of iterations, no matter what the initial conditions are. Also, a new pattern on the phase portrait is found, which has not been reported before. Finally, a conclusion is summarized in section 3 .

\section{Simulation Results}

According to our intensive simulations, we have the following observations:

\section{Observation 1.}

For the system described in [Chua, 1988, 1990; Galias, 1992; Kocarev, 1993, 1996;

$\mathrm{Wu}, 1993 ; \mathrm{Yu}, 2001]$, if

(i) $\quad b=-1$ and $|a|=2^{n}+\frac{1}{2^{n}}$ where $n \in \boldsymbol{Z} \backslash\{0\}$, or

(ii) $\quad b=a+1$ and $|b|>1$ and $a$ is an odd number, or

(iii) $\quad b=-a+1$ and $|b|>1$ and $a$ is an odd number,

then $\exists k_{0} \in \boldsymbol{Z}^{+} \bigcup\{0\}$ and $\exists M \in \boldsymbol{Z}^{+}$such that $\mathbf{x}(k+M) \approx \mathbf{x}(k)$ for $\forall k \geq k_{0}$ and $\forall \mathbf{x}(0) \in I^{2} \equiv\left\{\mathbf{x}(k):-1 \leq x_{1}(k)<1\right.$ and $-1 \leq x_{2}(k)<1$, where $\left.\mathbf{x}(k)=\left[\begin{array}{c}x_{1}(k) \\ x_{2}(k)\end{array}\right]\right\}$. 
Figure 1 shows the phase portraits of the system with $b=-1$, initial condition $\mathbf{x}(0)=\left[\begin{array}{l}0.1 \\ 0.2\end{array}\right]$, and with different values of $a$. It can be seen from the figure that the number of points in the phase portrait when $a=2.5$ or $a=-4.25$ is much less than that of the case when $a=3$ or $a=4$. One can verify that when $a=2.5$, $\mathbf{x}(k+214) \approx \mathbf{x}(k)$ for $\forall k \geq 163$, and when $a=-4.25, \mathbf{x}(k+54) \approx \mathbf{x}(k)$ for $\forall k \geq 191$. Because of this quasi-periodic property, the state vector $\mathbf{x}(k)$ will hit inside a very small neighborhood of the same point on the phase portrait after one period, so the phase portrait only shows a finite number of different points. However, there is no such quasi-periodic property when $a=3$ or $a=4$. Instead, there are infinitely many points on the phase portrait, and a random-like chaotic pattern is shown.

Similarly, Figure 2 shows the phase portrait and the symbolic sequences of such a system [Chua, 1988, 1990; Galias, 1992; Kocarev, 1993, 1996; Wu, 1993; Yu, 2001] with the same initial condition as those of Figure 1, but now $b=a+1$, and with different values of $a$. It can be seen from the figure that when $a=3$ or $a=5$, the symbolic sequences become zero and only finitely many points are on the straight lines of the phase portrait. One can verify that when $a=3, \mathbf{x}(k+2) \approx \mathbf{x}(k)$ for $\forall k \geq 28$, and when $a=5, \mathbf{x}(k+2) \approx \mathbf{x}(k)$ for $\forall k \geq 90$. On the other hand, there is no such quasi-periodic property when $a=4$. Hence, there are infinitely many points on those straight lines of the phase portrait. For $b=-a+1$, a similar result is obtained, as shown in Figure 3.

Is the above phenomenon true only for some particular initial conditions? We have conducted extensive simulations and find that it is true no matter what initial conditions are. Figure 4 shows the phase portrait of such a system [Chua, 1988, 1990; Galias, 1992; Kocarev, 1993, 1996; Wu, 1993; Yu, 2001] with $b=-1, a=2.5$, and with different initial conditions generated by a random number generator. It can be verified that the state vector $\mathbf{x}(k)$ converges to a periodic orbit for those initial conditions. And, similar results have been obtained for the case when $b=a+1, a=3$, or $b=-a+1, a=-3$, as shown in Figures 5 and 6, respectively.

\section{Observation 2.}

For the system described in [Chua, 1988, 1990; Galias, 1992; Kocarev, 1993, 1996; 
$\mathrm{Wu}, 1993 ; \mathrm{Yu}, 2001]$, if $b=-1$ and $|a|=2^{n}+\frac{1}{2^{n}}$ where $n \in\{-3,-2,-1,1,2,3,4\}$, then a new pattern, which looks like a rotated letter ' $\mathrm{X}$ ', is exhibited on the phase portrait, no matter what the values of the initial conditions are. The center of the rotated letter is located at the origin, and the slopes of the 'straight lines' of the rotated letter ' $\mathrm{X}$ ' are equal to the values of the pole locations.

Figures 1 and 4 show examples in which a rotated letter ' $\mathrm{X}$ ' is exhibited on the phase portrait centered at the origin and with slopes of the 'straight lines' being the values of the pole locations.

\section{Conclusion}

In this letter, we report that for some filter parameters in the extended boundaries of the stability triangle, the state vector will converge to a periodic orbit after a number of iterations, no matter what the initial conditions are. Also, a new pattern, which looks like a rotated letter ' $\mathrm{X}$ ', is found. The center of the rotated letter is located at the origin, and the slopes of the 'straight lines' of the rotated letter are equal to the values of the pole locations.

\section{Acknowledgement}

The work described in this letter was substantially supported by The Hong Kong Polytechnic University. 


\section{References}

[1] Chua, L. O. \& Lin, T. [1988], "Chaos in digital filters," IEEE Transactions on Circuits and Systems, vol. 35, no. 6, pp. 648-658.

[2] Chua, L. O. \& Lin, T. [1990], "Fractal pattern of second-order non-linear digital filters: a new symbolic analysis," International Journal of Circuit Theory and Applications, vol. 18, pp. 541-550.

[3] Galias, Z. \& Ogorzalek, M. J. [1992], "On symbolic dynamics of a chaotic second-order digital filter," International Journal of Circuit Theory and Applications, vol. 20, pp. 401-409.

[4] Kocarev, L. \& Chua, L. O. [1993], "On chaos in digital filters: case $b=-1$," IEEE Transactions on Circuits and Systems-II: Analog and Digital Signal Processing, vol. 40 , no. 6 , pp. 404-407.

[5] Kocarev, L., Wu, C. W. \& Chua, L. O. [1996], "Complex behavior in digital filters with overflow nonlinearity: analytical results," IEEE Transactions on Circuits and Systems - II: Analog and Digital Signal Processing, vol. 43, no. 3, pp. 234-246.

[6] Wu, C. W. \& Chua, L. O. [1993], "Properties of admissible symbolic sequences in a second-order digital filter with overflow non-linearity," International Journal of Circuit Theory and Applications, vol. 21, pp. 299-307.

[7] Yu, X. \& Galias, Z. [2001], "Periodic behaviors in a digital filter with two's complement arithmetic," IEEE Transactions on Circuits and Systems-II: Analog and Digital Signal Processing, vol. 48, no. 10, pp. 1177-1190. 

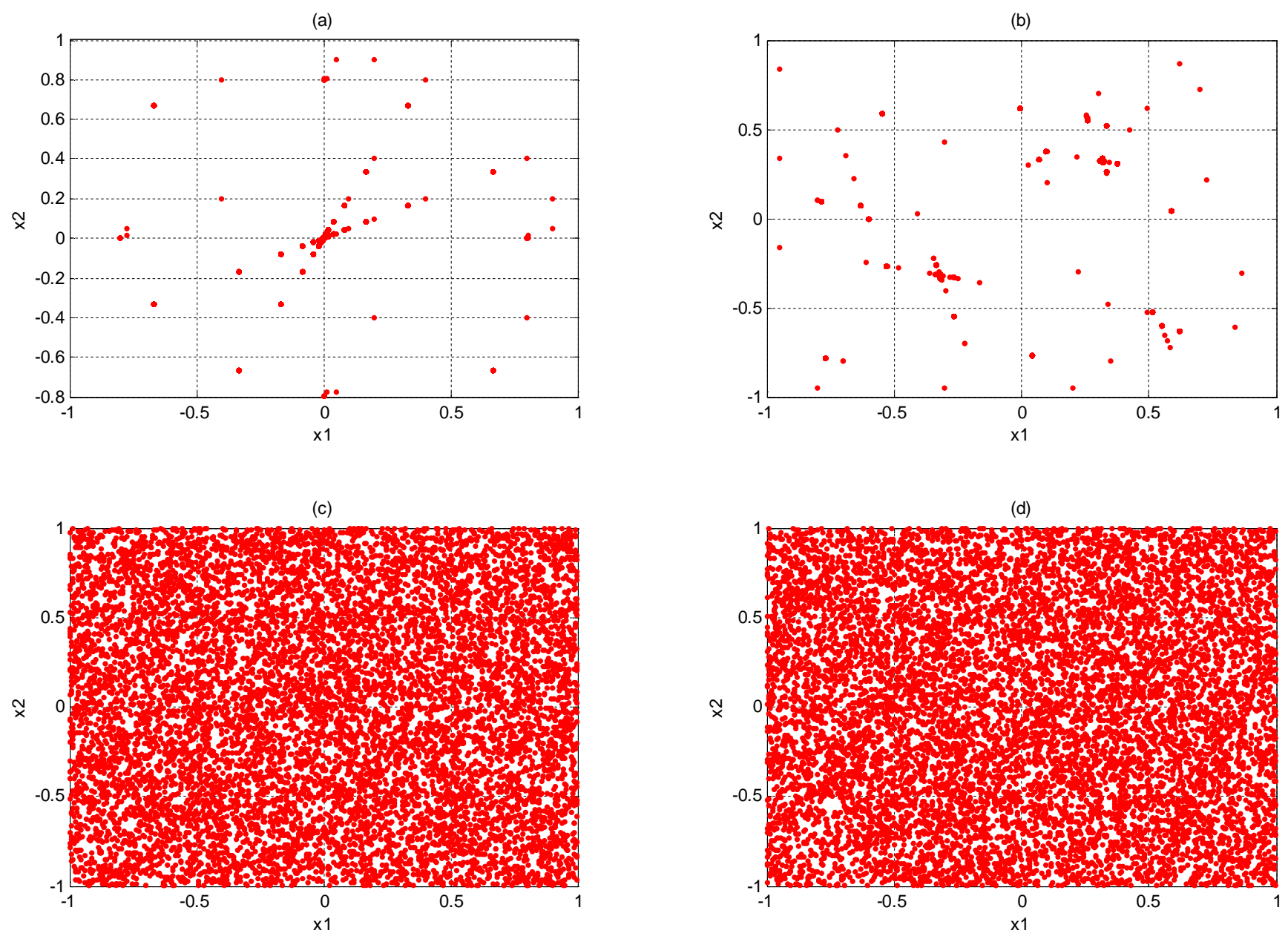

Fig. 1. Phase portrait with $b=-1, \mathbf{x}(0)=\left[\begin{array}{ll}0.1 & 0.2\end{array}\right]^{\mathrm{T}}$, and different values of $a$. (a) $a=2.5$, (b) $a=-4.25$, (c) $a=3$, (d) $a=4$. 


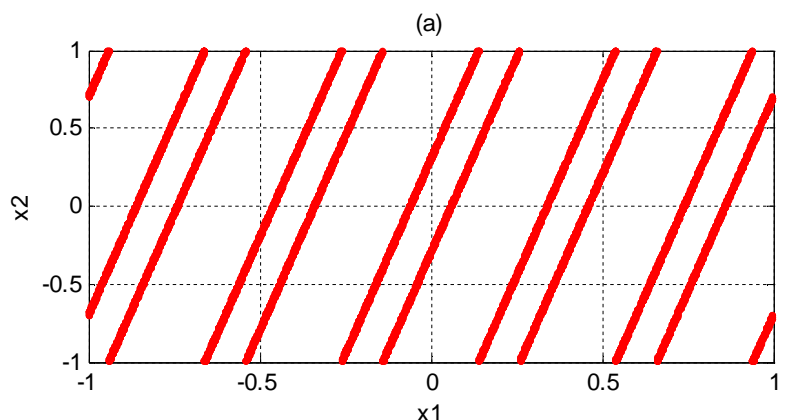

$x 1$
$(c)$



(e)



(b)

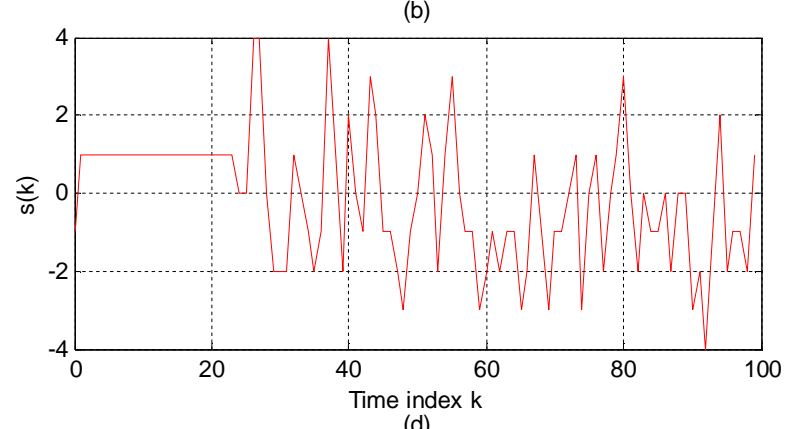

(d)

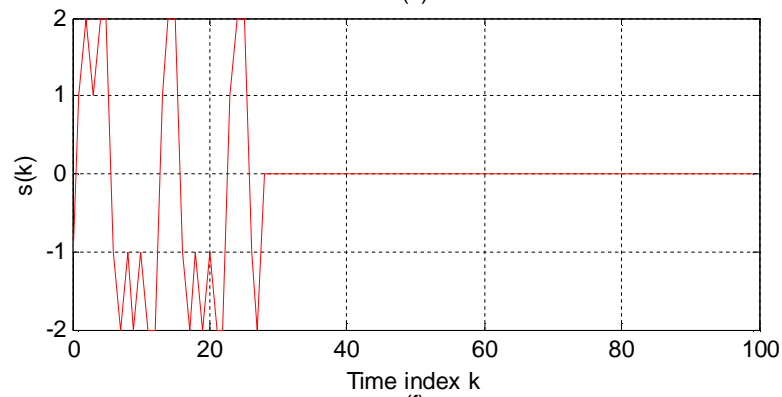

(f)

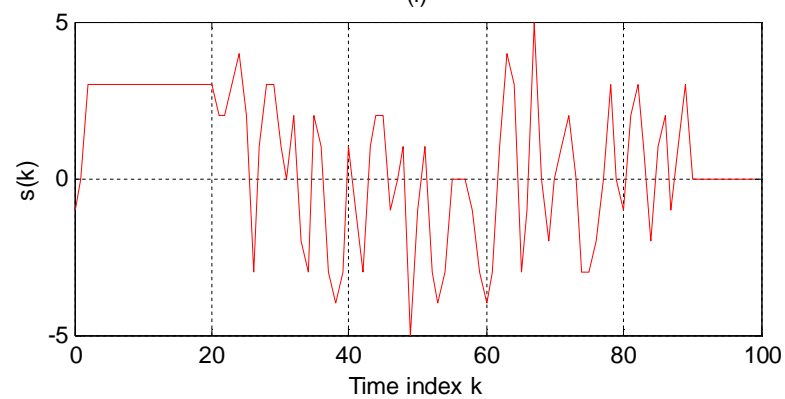

Fig. 2. Phase portrait and symbolic sequences with $b=a+1, \mathbf{x}(0)=\left[\begin{array}{ll}0.1 & 0.2\end{array}\right]^{\mathrm{T}}$, and different values of $a$. (a) phase portrait with $a=4$, (b) symbolic sequences with $a=4$, (c) phase portrait with $a=3$, (d) symbolic sequences with $a=3$, (e) phase portrait with $a=5$, and (f) symbolic sequences with $a=5$. 



(b)

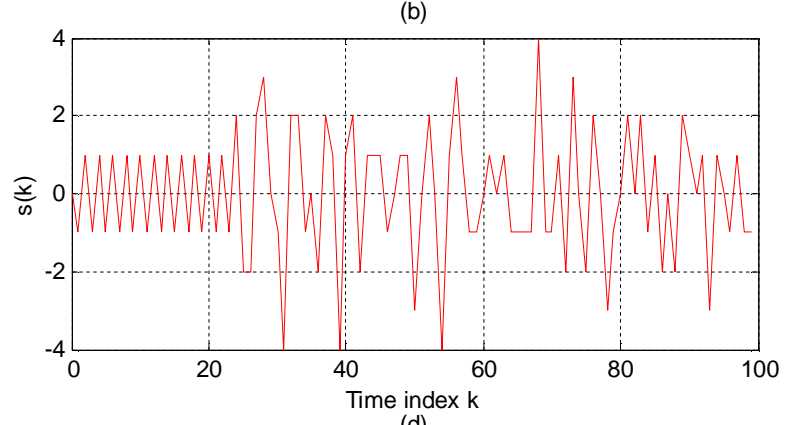

(d)

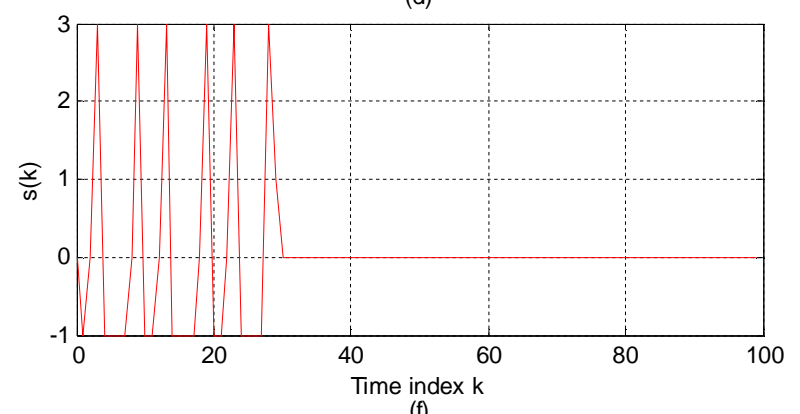

(f)

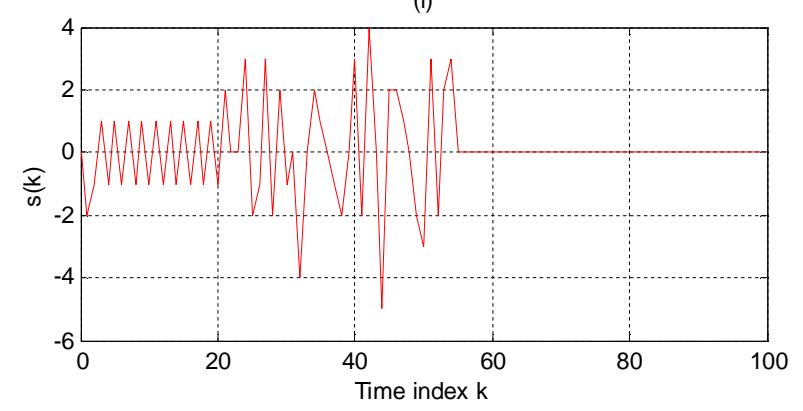

Fig. 3. Phase portrait and symbolic sequences with $b=-a+1, \mathbf{x}(0)=\left[\begin{array}{ll}0.1 & 0.2\end{array}\right]^{\mathrm{T}}$, and different values of $a$. (a) phase portrait with $a=-4$, (b) symbolic sequences with $a=-4$, (c) phase portrait with $a=-3$, (d) symbolic sequences with $a=-3$, (e) phase portrait with $a=-5$, and (f) symbolic sequences with $a=-5$. 



Fig. 4. Phase portrait with $b=-1, a=2.5$, and different initial conditions. (a) $\mathbf{x}(0)=[-$ $0.7222-0.4556]^{\mathrm{T}}$, (b) $\mathbf{x}(0)=[-0.5945-0.6024]^{\mathrm{T}}$, (c) $\mathbf{x}(0)=[-0.6026-0.9695]^{\mathrm{T}}$, and $(\mathrm{d})$ $\mathbf{x}(0)=\left[\begin{array}{lll}0.2076 & 0.4936\end{array}\right]^{\mathrm{T}}$. 
(a)
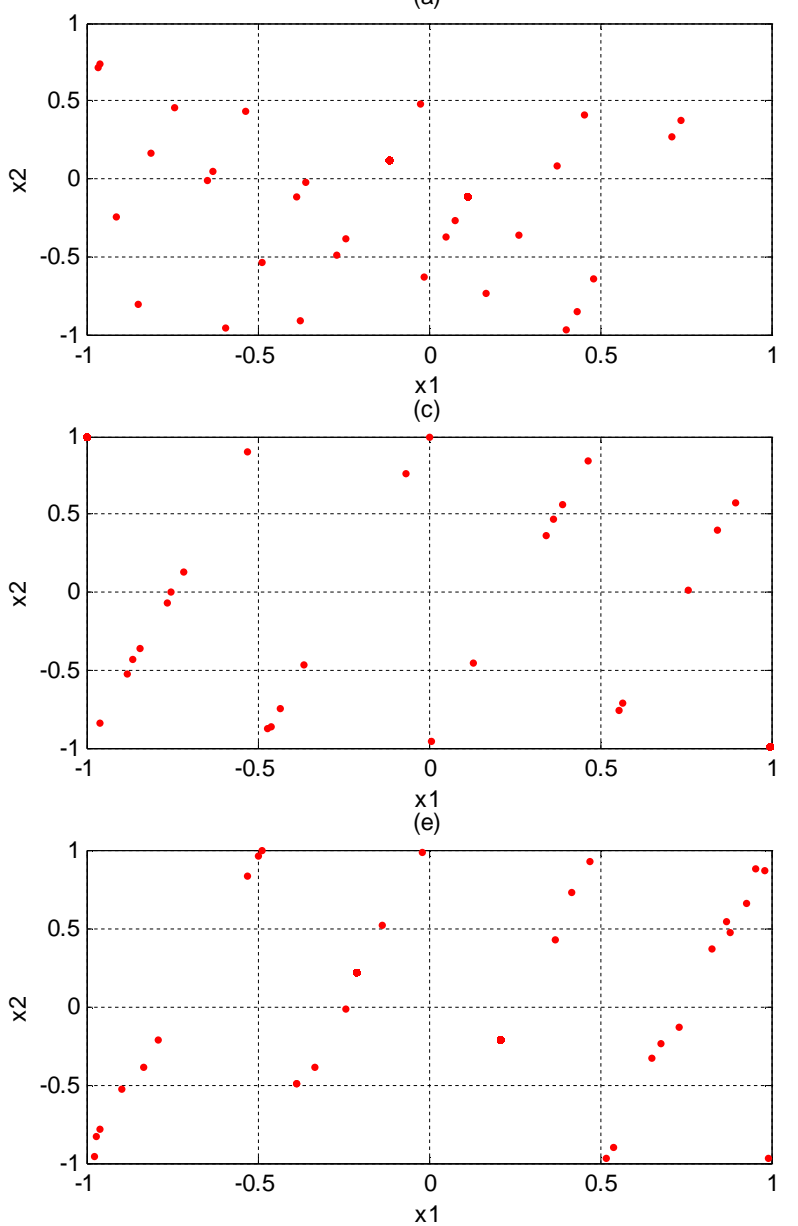

(b)

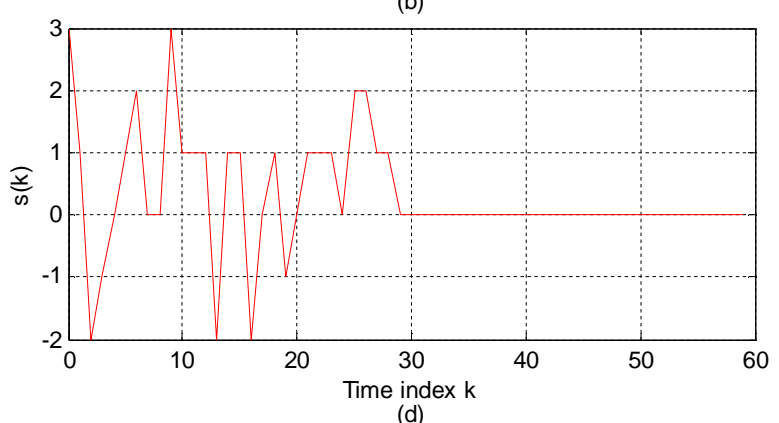

(d)



(f)

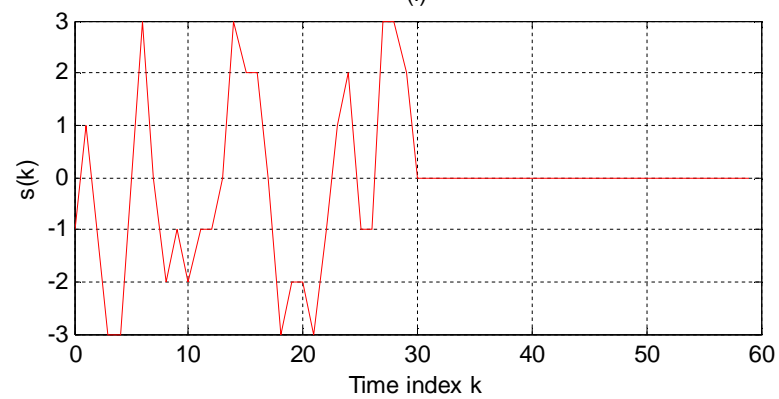

Fig. 5. Phase portrait with $a=3, b=a+1$, and different initial conditions. (a) $\mathbf{x}(0)=[-$ $0.5947-0.9607]^{\mathrm{T}}$, (b) $\mathbf{x}(0)=\left[\begin{array}{lll}0.3443 & 0.3626\end{array}\right]^{\mathrm{T}}$, and (c) $\mathbf{x}(0)=\left[\begin{array}{lll}0.6762 & -0.2410\end{array}\right]^{\mathrm{T}}$. 


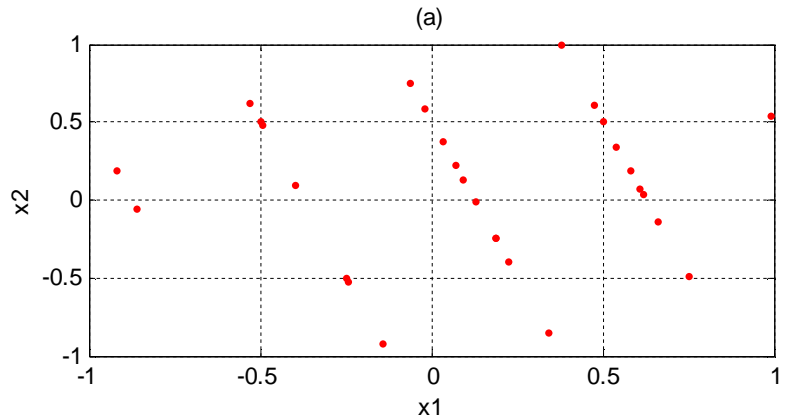

(c)



(e)

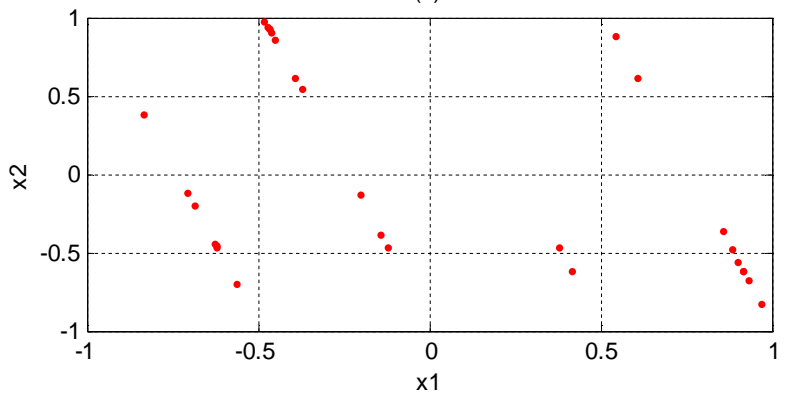

(b)

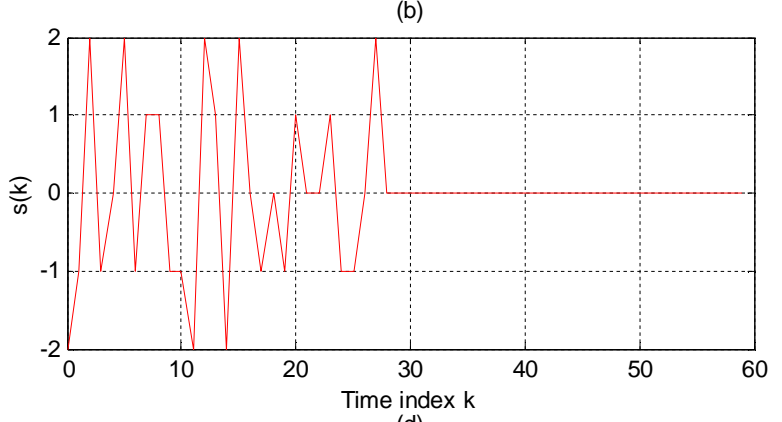

(d)



(f)



Fig. 6. Phase portrait with $a=-3, b=-a+1$, and different initial conditions. (a) $\mathbf{x}(0)=[0.6636-0.1422]^{\mathrm{T}}$, (b) $\mathbf{x}(0)=[0.0056-0.3908]^{\mathrm{T}}$, and (c) $\mathbf{x}(0)=[0.4189-0.6207]^{\mathrm{T}}$. 